\title{
A HYBRID INVENTORY CONTROL SYSTEM APPROACH APPLIED TO THE FOOD INDUSTRY
}

\author{
David Claudio \\ Jie Zhang \\ Ying Zhang \\ The Harold and Inge Marcus Department of Industrial and Manufacturing Engineering \\ Pennsylvania State University \\ University Park, PA 16802, USA
}

\begin{abstract}
The appropriate production and inventory control policy is a key factor for modern enterprises' success in competitive environment. In the food industry, most of food manufacturers adopt the make-to-order policy to improve their punctuality and flexibility, while some scholars provided other different opinions. This study provides a hybrid policy combining a make-to-order push strategy with priority with a make-to-stock pull strategy. In this policy, the pull strategy is considered for the regular demands while customers who tell their demand needs in advance are treated with a push strategy and are given a higher priority than those who don't share information. Through a set of simulation experiments by AutoMod model, this policy is proved to be of great efficiency, effectiveness and applicability.
\end{abstract}

\section{INTRODUCTION}

In modernized society, enterprises in the food industry are working in a very competitive environment, especially for the products of the traits of high perishability and fluctuation. In order to become order winners, it is very crucial for the firms to attack the issues of products costs, on-time delivery and flexibility. Therefore, most of the food manufacturing firms adopt a make-to-order strategy. However, if the lead time of a make-to-order process is longer than the delivery time requested, a make-to-stock strategy is preferred (Hopp and Spearman, 1996). Reiner and Trcka (2004) discussed some alternatives for a production company in the food industry based on simulation. In one section of their paper they discuss a pasta production facility with multiple products. For this problem they have chosen to simulate the production process as a make-to-stock process with a reorder point heuristic under continue review. According to them, make-to-stock is the standard production strategy for the pasta producer. Reorder point policy is one approach for controlling inventory while maintaining a desired service level. When inventory is monitored continuously, the reorder point policy consists on placing an order of size $\mathrm{Q}$ when the inventory level reaches or goes below a reorder point, $r$ (Hopp and Spearman 1996). This is usually referred to as a (Q,r) model. This policy, while maintaining high service levels, usually incurs in high cost of carrying inventories.

Our objective in this paper is to simulate a scenario similar to the one described by Reiner and Trcka (2004) and to investigate the benefits on changing the strategy they used in their paper. Rather than just using the $(\mathrm{Q}, \mathrm{r})$ reordering strategy we are proposing a hybrid strategy between pull and push systems with priorities on customers who share information in advance.

Pull systems have been successfully implemented in several industries. Among the most pull-type production and inventory control systems (PICS) we find the kanban system, and CONWIP. In a pull system, all customer demands are expected to be satisfied from stock, hence when a customer order arrives, the probability of finding a product is high and the service levels can be maintained above a desired point. In addition, the time the customer has to wait for the product to be shipped is zero if the product is available. For this we need to be operating under a maketo-stock policy, which might yield high costs due to carrying inventory. According to Hopp and Spearman (1996) push systems are inherently make-to-stock while push systems are make-to-order. That is, the schedule that drives a push system is driven by orders (or forecasts) and not by system status.

The make-to-order policy can reduce the costs incurred in carrying inventories at the cost of reducing the service levels and customers having to wait for their products to be manufactured. Perhaps the most famous maketo-order strategy is MRP which works better when customer demands are known in advanced or when variability is demand forecasts are relatively low.

Many people argue that in order to improve operational efficiencies, supply chain partners need to start looking for new ways to collaborate. Reiner and Trcka (2004) 


\section{Claudio, Zhang and Zhang}

mention that the information-sharing policy of the retail chain companies is very restrictive. But recent developments in information technology and the emphasis on supply chain system integration have significantly reduced the cost of obtaining end-item advance demand informationhenceforth referred to as ADI - in the form of actual orders, order commitments, forecasts, etc., and diffusing it among all stages of the system (Liberopoulos and Koukoumialos, 2005).

Our proposed system is a hybrid model that combines a make-to-order push strategy with information sharing with a make-to-stock pull strategy. The make-to-stock strategy will be applied to those customers who come in requesting a product at the time of arrival, and the maketo-order will be applied to those who can give information in advance about their needs. These customers will have a higher priority than the ones that do not share information about their needs. With our proposed strategy we might not have the full benefits of controlling the total work in process (WIP) material or the total finished goods (FG) inventory, but we have partial benefits, as we can control the WIP and FG inventory of the customers that do not give information in advance, to whom we have labeled as "regular customers". In addition, the time in the FG inventory for customers who share information stays in the FG area is minimal since the purpose of sharing information is to have the product just in time for when the customer expects its product to be shipped. In other words, with information sharing applied to a maketo-order strategy we can obtain the benefit of reducing inventories but without having to pay the cost of lowering the service levels or customers' waiting for their products. At the same time, for customers who do not share information we keep some FG in order to also increase the probability of they finding the product just in the time for when they request it.

The importance of this paper is that we are proposing a variation of the already proposed systems that integrate information sharing. In the next sections we will discuss in more detail what are the variations that make our approach different than the already proposed strategies.

The rest of the paper is organized as follows. Section 2 provides a brief review of the literature. Section 3 describes the proposed strategy as well as the assumptions made for the study. Section 4 provides a description design of experiments. Section 5 provides a summary of the results obtained for systems operating under hybrid strategy. Section 6 provides a summary of the results and discusses extensions of the current research.

\section{LITERATURE REVIEW}

Production and inventory control systems such as MRP systems, and kanban control systems (KCS) have been the subject of intensive research for several years. There are some excellent summaries of how to design a manufactur- ing plant under these systems and the issues and benefits gained from such systems. They can be found in literature such as Buzacott and Shanthikumar (1993), Hopp and Spearman (1996) and Zipkin (2000). Pull systems are the easiest to implement and yet very efficient. Kanban control systems explicitly limit inventories using kanbans (cards) when one unit is consumed from inventory, a kanban signal is sent upstream to begin work to replenish this inventory (Krishnamurthy and Claudio, 2005).

Prior research such as that reported in Hopp and Spearman (1996) have shown that limiting inventories and triggering production in this manner yields better system performance when compared to traditional MRP/push systems. Alternative pull systems such as the CONWIP system (Hopp and Spearman, 1996) also have similar advantages. Some of these studies also suggest that in manufacturing environments with high product variety pull systems could be inefficient (Krishnamurthy, Suri, and Vernon 2004).

One approach to overcome the disadvantages of pull systems has been to focus on how best to combine JIT and MRP philosophies in order to maximize the benefits of both strategies. A hybrid production system could be characterized as a production system that combines elements of the two philosophies in order to minimize inventory and unmask flaws in the system while maintaining the ability of the system to satisfy demand (Geraghty and Heavey, 2005). One example is the Synchro MRP, developed by Yamaha Motor Company, which utilizes MRP for long range planning and Kanban control system for shop floor execution. The problem with Synchro MRP is that it links MRP into every work center, resulting in high control complexity (Beamon and Bermudo, 2000).

Over the past years, new hybrid systems have been developed incorporating the use of the information sharing between customers and suppliers. This information sharing is commonly defined as advance demand information (ADI). Karaesmen, Buzacott, and Dallery(2002) consider a particular type of ADI also referred to as advance order information: early commitments on orders from customers. Krishnamurthy and Claudio (2005) and Liberopoulos and Koukoumialos (2005) studied the effects of ADI in pull strategies.

The strategy we propose is similar to the one discussed in Liberopoulos and Koukoumialos (2005) in that it considers ADI. The difference is that in the hybrid system described by them, the ADI is part of a joint station where it needs the synchronization of three items in order to release a production signal: the kanban card, the raw material, and the order. In our proposed strategy we do not need a kanban card to match the advance orders.

In their experiments, Krishnamurthy and Claudio (2005) found that in a typical pull system, such as the simple kanban system, if the kanban cards are allocated at the finished goods inventory area, the whole purpose of know- 


\section{Claudio, Zhang and Zhang}

ing an order in advance is diminished since the order will arrive in advance and will wait at the fork/joint stage until a kanban card arrives, which will happen when the customer arrives and grabs one unit of inventory. Liberopoulos and Koukoumialos (2005) also describe this effect by stating that the kanban policy cannot exploit ADI, because in the kanban policy a production order is placed after a part in FG inventory is consumed and therefore at (or after) the due date of the demand that triggered it. In addition, Krishnamurthy and Claudio (2005) also found that the benefit of having ADI resides in lowering the inventories while keeping the same amount of kanban cards, but allocating them at the beginning of the first station. This way, when the order arrives in advance, it can match the raw material and the kanban card and send a signal to start producing one item. Liberopoulos and Koukoumialos (2005) described such system where kanban cards are allocated at the beginning of the first station and presented the singlestage hybrid base stock/kanban policy with ADI. Here, the system starts with a base stock of $\mathrm{S}$ end-items in FG inventory and $\mathrm{K}$ free kanbans. Where the number of free kanbans represents the number of parts that can be released into the facility before the WIP in the system reaches the WIP-cap level K (Liberopoulos and Koukoumialos, 2005).

In that sense, as previously mentioned, our proposed system is different than the previously described in that not all orders need to be matched to a kanban card so that it can be released for production. Hence, our system is a hybrid in the context that it is a combination of pull and push strategies acting at the same time. The system will behave as a CONWIP as described by Hopp and Spearman (1996) for regular customer orders that arrive and expect to find the product. These customers have no guarantee that the product will be there and if there is no product they will be backordered. The maximum inventory for this type of customers at any point is given by the number of kanban cards in the whole system, K. At the same time, the system will behave as a push system when order comes in advance. When this happens, the order will be released for production according to Karaesman et al. (2002), where two delay node holds the order for: $\max (0, \tau-\mathrm{LT})$, and $\tau$, where $\tau$ is the amount of advance time the customer gave the supplier, and LT is the manufacturing lead time. These orders do not need a kanban card to be released for production and will have a higher priority in both the manufacturing process and at the FG area as an incentive to the customer for giving the order in advance. Thus, a customer that notifies his requirements in advance will have the benefit that the order will be processed before any regular order at any work station to ensure that the order will be at the FG inventory by the time it needs to be shipped. In addition, if by the time the order needs to be shipped the order is not ready, since the product for the regular orders and the advance orders is the same, the order will be replenished from the regular inventory and shipped to the advance customer and the kan- ban cards will simply be detached and will be attached to the items that corresponded to that order once they are released from the production cycle. The strategy will be described in more detailed in the coming sections.

\section{SYSTEM DESCRIPTION}

\subsection{Description Of Our Proposed System}

Since we are taking into account a manufacturing system similar to the one discussed in Reiner and Trcka (2004), let us consider a system with two manufacturing stages in series (denoted by $\mathrm{M}_{\mathrm{i}} \mathrm{i}=1,2$ ) as displayed in figure 1 . The first stage consists of production of the food, where in the second stage the packaging takes place. Two types of orders may arrive to the supplier. The first one is just demand orders from those customers who request an item and expect the order to be shipped at that moment. We refer to these as regular orders. The second type of order is the one where customers tell the supplier in advance their requirements. We refer to these as advance orders.

Since we want to minimize the waiting time of the customer, regular orders will be handled in a make-tostock strategy. The inventory kept for regular orders is limited by the total number of kanban cards, denoted by $\mathrm{K}$.

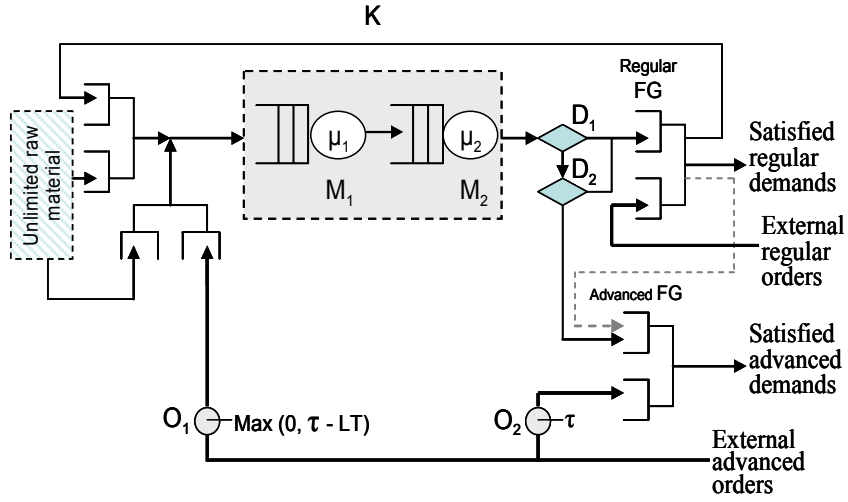

Figure 1: Proposed hybrid push/pull system

When regular order arrives, if there is at least one item in the finished goods (FG) buffer, then it is used to satisfy the order. At the same time, the kanban card attached to the item is sent back to the beginning of the manufacturing stage where it will enter a fork/joint station waiting to match with a raw material in order to authorize production. For purposes of this study we will assume the kanban card signals the production of one item. We also assume unlimited supply of raw materials. The kanban card together with the raw material queue for its turn at stage 1 and sequentially get processed in stage 1 and 2 . After getting out of the manufacturing process, the product will enter a decision node where it asks if the product corresponds to a regular order or to an advance order and join its respective FG 


\section{Claudio, Zhang and Zhang}

area. If upon order arrival of a regular order, there are no items in the FG buffer, it is backordered and the order waits until an item is available. This part of our proposed strategy ensures the number of units in the finished goods inventory for regular orders at most equal to $\mathrm{K}$.

For the advance orders, we assume that each customer places an order with the manufacturing facility $\tau$ time units in advance of their actual requirement (due date). We call $\tau$ the amount of time in advance the information was given. For simplicity, we assume that this time is constant and same for all customer orders. Furthermore, we assume that once an order is placed they will not be cancelled or changed. At the same time, the plant has a manufacturing lead time, denoted by LT, which corresponds to the lead time used by the system to plan production. The manufacturing lead time typically depends on the average time taken to actually manufacture a unit. Again, for simplicity a constant lead time has been assumed.

When an advance order is placed, it is duplicated and sent into two delay stations. At delay station O1, the manufacturing system introduces a delay $\mathrm{T} 1=\max (0, \tau-\mathrm{LT})$, between when an order is received and when it is activated upstream to trigger production via raw material release. This ensures that if the amount of units in advance, $\tau$, is less or equal to the manufacturing lead time, the order will be immediately released for production as discussed in $\mathrm{Ka}-$ raesman et al. (2002). Once the order is released from $\mathrm{O} 1$ after T1 time units have passed, the order will then join a fork/joint station where it will be matched with a raw material in order to be released for production. Note that, as mentioned before, this policy differs from others in the sense that it does not need to be matched with a kanban card. At the same time, the duplicate of the order sent to the delay station $\mathrm{O} 2$ will wait for $\tau$ units of time before been converted into actual demand and the customer will then expect his order to be shipped at that time. As mentioned before, advance orders will have a higher priority in both the manufacturing process and at the FG area, as an incentive to the customer for sharing information. Thus, a customer that notifies his requirements in advance has the benefit that his order will be processed before any regular orders at any work station to ensure that this order will be available at the FG inventory to be shipped at the requested time. If by the time the order needs to be shipped but it is not ready, since the product for the regular orders and the advance orders is the same, the order will be replenished from the regular inventory and shipped to the advance customer. And the kanban cards will simply be detached and will be attached to the items that corresponded to that order once they are released from the production cycle, as represented by the dotted line that comes out of the regular FG area into the advance order FG area. If there are no products in neither of the FG areas then the advance demand is backordered and will get the next product that comes out.
When a product comes out of the production cycle, it goes into the decision node D1. If the item belongs to an advance order then it will enter a second decision node, D2. Here it will be asked if the demand corresponding to that item has already been fulfilled. If it has, then it will go to the regular FG inventory where a kanban card should be waiting for it to be attached. If the answer is no, then it will continue to the advance FG area. In order to better explain the process, figure 2 contains a flow chart of the logic that occurs from the moment an order arrives. In the flowchart, when an order comes out of the packaging process, it asks if the order is an advance order (D1). If the answer is yes, then it continues to ask if $V_{-}$flag is bigger than zero (D2). $\mathrm{V}$ flag is our representation of the situation when an advance customer takes one product from the regular FG area. When this happens, a flag is raised (hence the name $\mathrm{V}$ flag) to point out that this situation has occurred and that there is at least one kanban card waiting for a product to be attached to. Note that this situation can happen many times within a time interval, thus we do not limit V_Flag to just a binary variable to denote it is raised or not since we need to keep track of how many times this has occurred.

With this strategy one of three situations might happen to the advance customer. Firstly, the product might reach the advance FG area before the advance demand does. In this case the inventory in the advance FG area will increase but only for a relatively short period of time since the demand should be released from delay node $\mathrm{O} 2$ shortly after this happens. In this sense, the advance FG inventory area can be seen as a temporary holding area until the order is shipped to the advance customer. The second situation arises when the demand is released from node $\mathrm{O} 2$ before the product reaches the FG area. In this case, as discussed before, the customer will get an item from the regular FG inventory and will not have to wait for his product. At the same time, the product should be arriving shortly so the kanban card should not be by itself for long. The third situation is when the advance demand arrives first and finds no inventory in either area. In this case the demand will be backordered but since the order is expected to arrive in a relatively short time, the customer will only have to wait for a small amount of time which is still better than having to wait for the whole manufacturing lead time. The benefits gained from this strategy can be found in the reduction of inventories and at the same time, the customer will not have to wait for its product (unless the previously discussed case three happens).

For our study, we have assumed that external customer orders (demand) arrive one at a time and are satisfied whenever possible from units available in the finished goods inventory buffer. If units are not available, customer requests are backordered and are satisfied immediately upon availability of inventory giving priority to the advance orders. 
Flowchart of our proposed strategy



Figure 2: Pull/push ADI flowchart 


\section{Claudio, Zhang and Zhang}

As previously mentioned, we have assumed that LT is constant as well as the amount of advance demand information, $\tau$, which will be the same for all customer advance orders. For simplicity, we have also assumed that $\tau$ is bigger or equal than LT.

We set demand to be normally distributed with mean two hour and standard deviation 20 minutes as suggested in the case presented by Reiner and Trcka (2004).

\subsection{Description of Simulation}

The strategy was simulated with Automod 11.1. At first we simulate the make-to-stock policy to obtain the general cycle time.

We use a process to generate the two kinds of demands which are called general and advance demands as can be seen in figure 2. A changeable variable representing the proportion of these two kinds of orders was set for the future analysis. For example, the proportion 9:1 refers to the case where $90 \%$ of the demand orders came from customers that gave information on advance while only $10 \%$ of the demand orders were from customers who gave no information in advance.

Subsequently, we use another process to schedule the flows for each kind of demand. For the general demand, it may be sent to process to cope with the backorders under the condition of no available inventory here, or otherwise send the signal to production to supplement the taken-off products. For the advance demand, we use a specific process to schedule the production to satisfy the advance order, such as the production time will be scheduled in a specific time so that the arrival of the customer could match the accomplishment of the production. In our case, the manufacturing station M1 has exponentially distributed service times with mean four hours, and station M2 has exponentially distributed service times as well with mean one hour. And for simplicity, it is assumed that the manufacturing stages have finite production capacities, implying that products could potentially queue in the buffers between the stages and wait for the resources in a manufacturing stage. In addition, it is also assumed that there is sufficient supply of raw material at the beginning of stage. Moreover, we also use a single process to break up and down the operating machines to simulate the reality.

\section{SETUP OF SIMULATION EXPERIMENTS}

In order to eliminate the potential initial effects, we set the warm-up period to 50 hours which was obtained by doing a steady state analysis.

AutoStat was used to analyze our experiments since it is strong statistical analysis software within AutoMod. With AutoStat we varied some of the factors and calculated the number of backorders to get the potential costs of losing customers, as well as the average time spent in the inventory by single goods multiplying the average number of goods in the stock to calculate the holding costs. In this case, we assume the proportion of backorder costs to the holding cost is 2 to 1 .

Using the "vary multiple factors" analysis in the AutoStat, we the proportion of advance demand to general demand which could represent the market demand situation, as well as the initial number of kanban cards, $\mathrm{K}$, which represents the maximum inventory for regular demand at any point in time. Table 1 contains the range for each of these variables.

Table 1: Range of variables

\begin{tabular}{|c|c|c|c|}
\hline Variables & maximum & minimum & step \\
\hline $\begin{array}{c}\text { Advance demand : } \\
\text { General demand propor- } \\
\text { tion }\end{array}$ & $9: 1$ & $1: 9$ & 1 \\
\hline maximum inventory (K) & 6 & 1 & 1 \\
\hline
\end{tabular}

\section{RESULTS}

Based on the Bonferroni principle we used 10 replicates for each simulation run. From the results, we conclude that the minimum costs can be found when the proportion of demand orders with ADI to regular demand is 5:5 (or 50\% for each), and the maximum inventory is 6 . Our strategy strives to get the best of both push and pull systems combined and the optimal point can be found when both strategies are equally implemented, in other words, when the system is not behaving more like one strategy or the other. It is reasonable because if the proportion of advance demand is higher then the time orders holding the regular inventory will be longer, which will cause a higher inventory holding cost; if the proportion of regular demand is higher then the fluctuation will be greater, which will make the general backorders higher. So a trade-off happens at the point of fifty-fifty.

In addition, using the "compare all to one" function in the AutoStat, we could also confirm our conclusion. From table 2 we could tell that the systems, in which the proportion of ADI and regular demand is 6:4 with other variables at the same value, is not statistically distinguishable.

Table 2: CI for the costs differences

\begin{tabular}{|c|c|c|c|c|}
\hline $\begin{array}{c}\text { Advance demand : Gen- } \\
\text { eral demand proportion }\end{array}$ & $5: 5$ & $6: 4$ & $7: 3$ & $8: 2$ \\
\hline Maximum inventory & 6 & 6 & 6 & 6 \\
\hline average cost difference (\$) & 5.44 & 3.09 & 5.34 \\
\hline CI low for cost difference & 2.88 & 1.34 & 2.63 \\
\hline CI High for cost difference & 0.32 & -0.40 & -0.08 \\
\hline
\end{tabular}




\section{Claudio, Zhang and Zhang}

Therefore, we could conclude that our proposed system is also robust when ADI is relative higher. However in order to get a deeper sense of the impact of different proportion and maximum inventory, we refer to the Excel to do the three dimension surface plots.

From figure 3, we can see that there is a tradeoff between the demand proportion and the number of kanban cards. Thus, in order to reduce costs we need to either increase the number of kanban cards or increase the proportion of advance demands. At kanban number of 6 we see that the advance demand information have no significant effect. This means that with 6 kanban cards we do not need to have ADI in place, but on the other hand, if we wanted to reduce our inventories, then we could for example, reduce it to 4 and incrementing the proportion of ADI to $50 \%$. In addition, from figure 4 , we find that with the increase of ADI, the total number of backorders will decrease to some extent. That information is very important because it shows that using our proposed system could efficiently reduce the number of backorders, which is good news to hold the customers for the manufacturers. On the other hand, the number of backorders for the advance demand will dramatically increase when the proportion of this kind of demand increase, as shown in figure 5.

Taking a closer look into the backorders and the FG inventories, we can see in figure 5 that the total number of backorders for the advance orders is dependent on both, the number of kanban cards and the proportion of advance orders to regular orders. This makes sense since the lower the number of kanban cards, the more the strategy will behave as a simple push system.

From figure 6 we can see that now the backorders for the regular demand depends on the interaction of both, the number of kanban cards and the demand proportion. If the proportion of regular demands is very small, as in the case 9 (meaning 9 advance orders to 1 regular order), then it will not matter that how many kanban cards we have because the number of order coming is very small. In a certain way, this is the purpose of including some orders with ADI. Conversely, when the proportion of regular demands is high, then we should increase the number of kanban cards to reduce the number of backorders. Finally we can also see the average time spend in these two inventories for the finished products (figure 7), from which we could conclude that if we want to reduce the average stocking time for the products for the general demand, we should then decrease the number of kanban cards. On the other hand, decreasing the number of kanban cards could do nothing to reduce the average storing time in the inventory for the advance demand as seen in figure 8 . Figure 9 plots the average number of advance inventory versus the number of kanban cards for each set of proportions. Since the advance orders do not depend on the number of kanban cards, the inventory is pretty much affected by the proportion and not by the number of kanban cards.

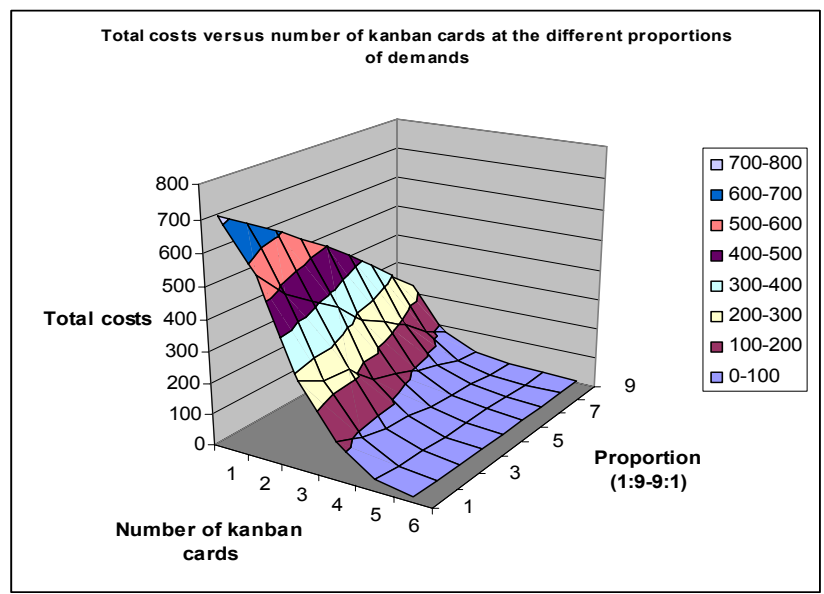

Figure 3: Surface plot for the total cost based on demand proportion and kanban cards

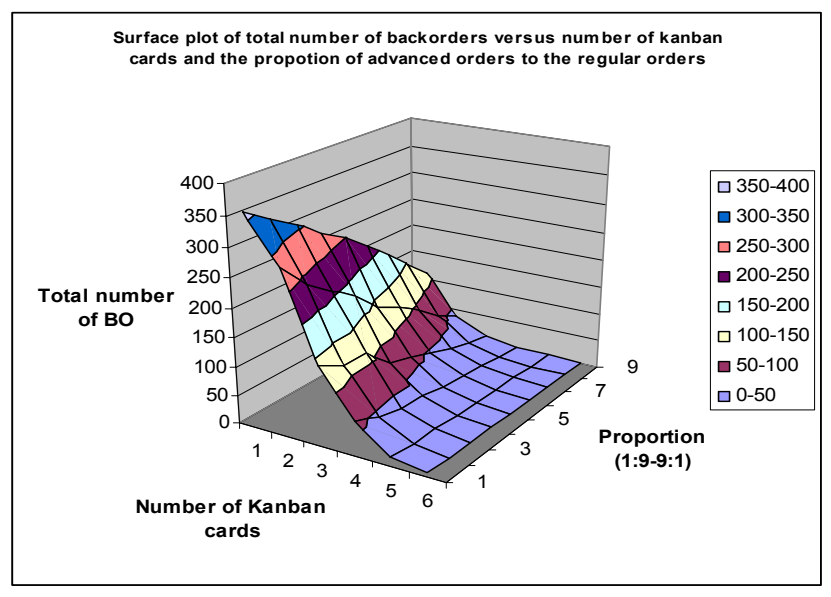

Figure 4: Surface plot for total number of backorders based on demand proportion and kanban cards

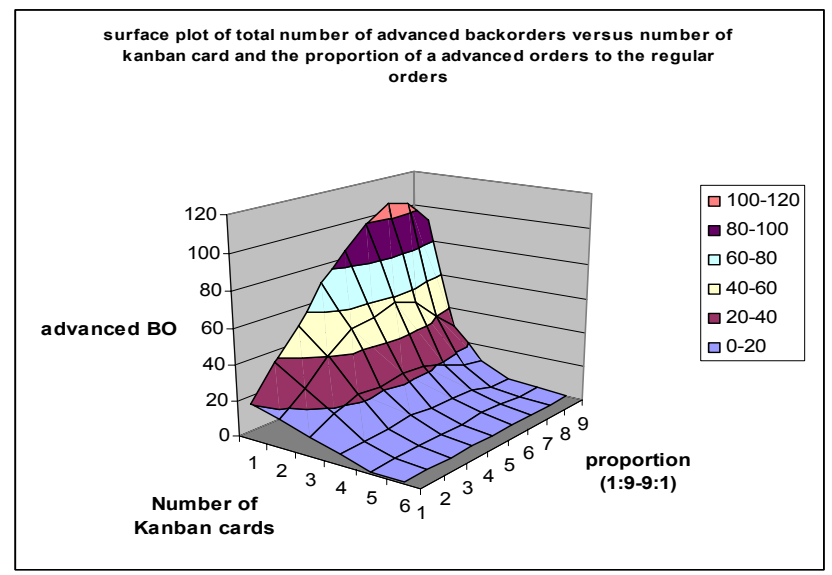

Figure 5: Surface plot for number of backorders for the advance demand based on demand proportion and kanban cards 




Figure 6: Surface plot of total number regular backorders based on demand proportion and kanban cards

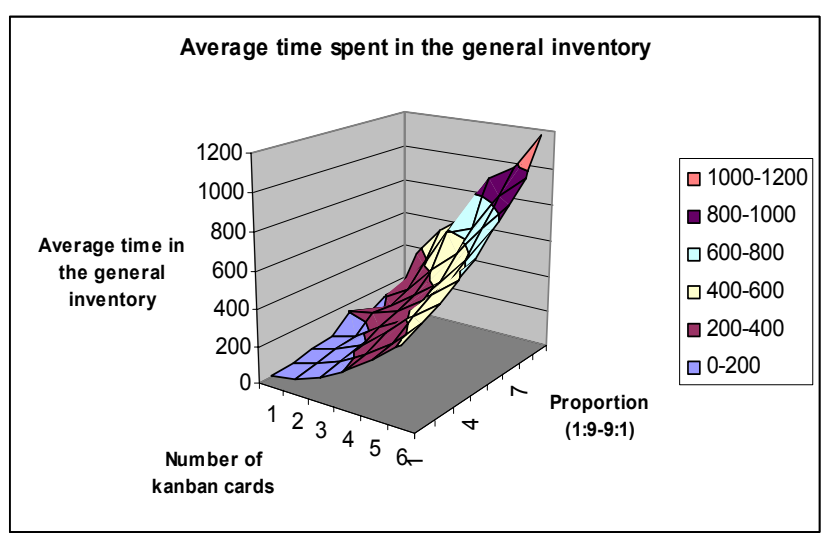

Figure 7: Surface plot of average storing time spent in the general inventory

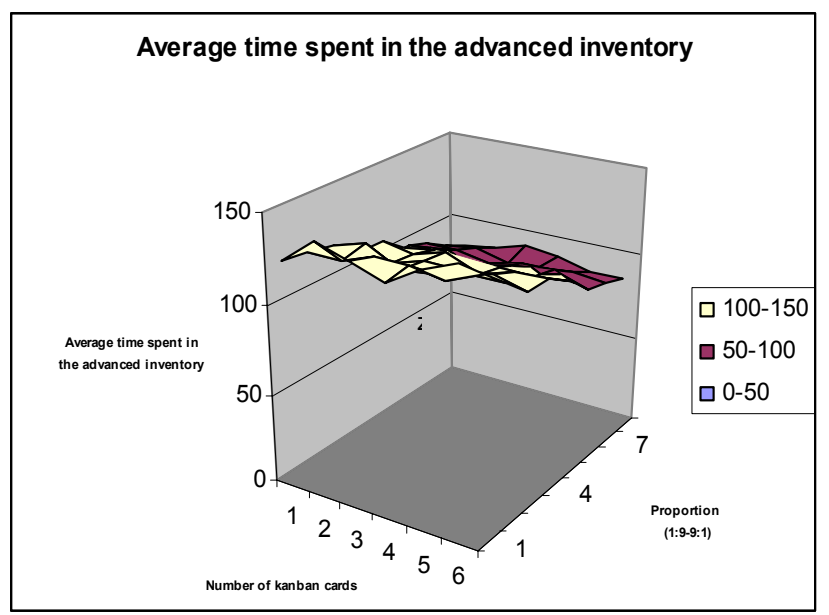

Figure 8: Surface plot of average storing time spent in the inventory for the advance demand

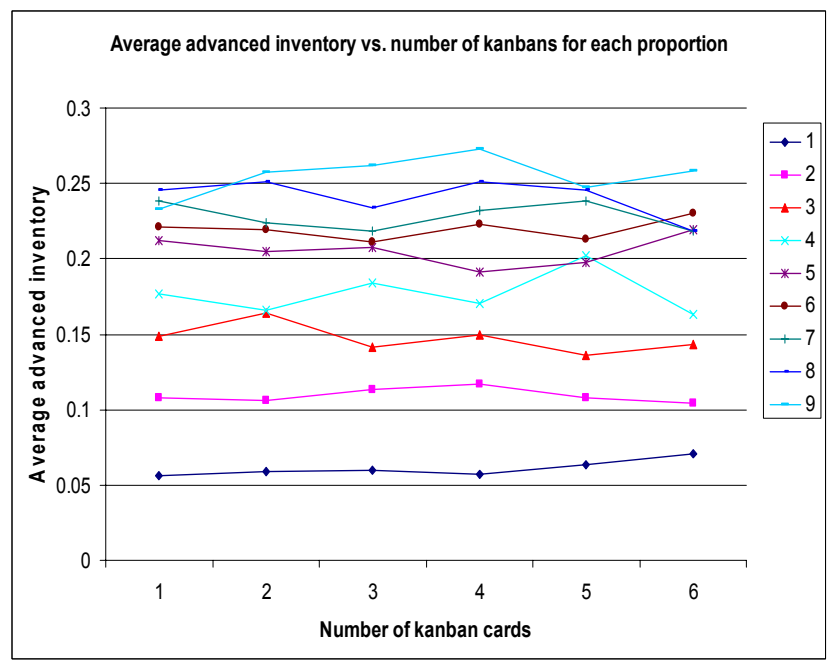

Figure 9: Average number of advance inventory vs. the number of kanban cards for each proportion

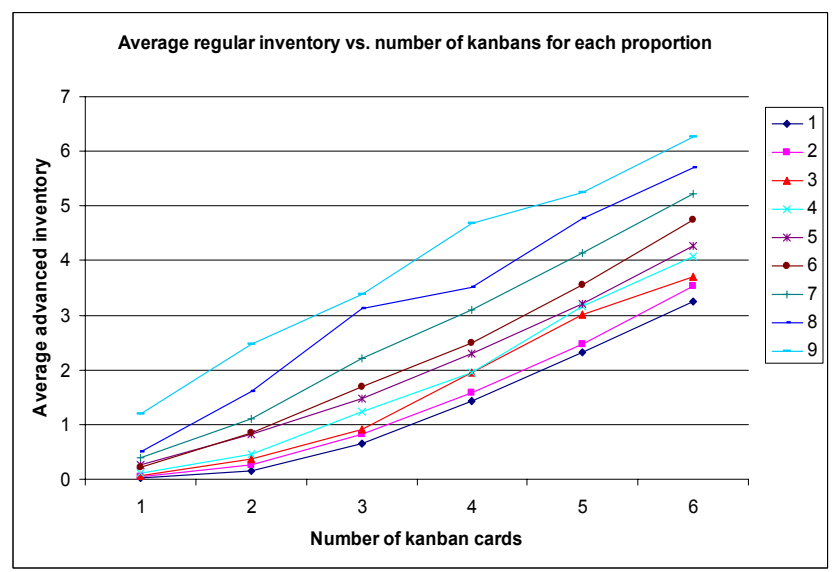

Figure 10: Average number of regular inventory vs. the number of kanban cards for each proportion

In addition we can see that, as predicted when we proposed the system, this number is not even greater than 0.3 units, which means that having ADI does not significantly increase the number of units in inventory.

Figure 10 plots the average number of regular inventory versus the number of kanban cards for each set of proportions. Here we can see an almost linear relationship between the number of kanban cards and the average inventory.

This part of our strategy is almost behaving completely like a regular pull system in the sense that the maximum inventory does not exceed the number of kanban cards, with the exception of when the regular orders are only $10 \%$ of the demand; the average inventory seems to exceed the number of kanban cards. This might be due to the interaction that exists between the two strategies working together (e.g. the decision nodes previously explained). 


\section{Claudio, Zhang and Zhang}

\section{SUMMARY AND CONCLUSIONS}

In this paper we propose a hybrid system, which combines a make-to-order push system with a make-to-stock pull system with information sharing, depending on the nature of the order. The make-to-stock strategy will be applied to those customers who come in requesting a product at the time of arrival, and the make-to-order will be applied to those customers who can give information in advance about their needs.

After a some analysis of our proposed system, we find that it serves the purpose of looking for a tradeoff between reducing the inventory levels and the service levels( as seen by the backorders). We found the best parameters within our system, which were the ones that minimized the total costs happened to be at a demand proportion level of $50 \%-50 \%$ between demand orders with information sharing and those with no information in advanced.

However there are also some insufficiencies in this paper. Future research might include the comparison of this strategy to other existing strategies such as kanban, CONWIP, POLCA, MRP, among others. In addition, derivation of analytical models to describe the system might prove to be helpful to better understand the effects of varying some of the system parameters. Some extensions might be included to analyze the behavior of the strategy when the manufacturing lead-time is not constant, and to analyze the strategy in a multi-product environment.

\section{REFERENCES}

Beamon, B., and J. Bermudo. 2000. A hybrid push/pull control algorithm for multistage, multi-line production systems. Production Planning \& Control 11(4):349356.

Buzacott, J. A., and J. G. Shanthikumar. 1993. Stochastic Models of Manufacturing Systems. Englewood Cliffs, NJ: Pretice Hall.

Geraghty, J., and C. Heavey. 2005. A review and comparison of hybrid and pull-type production control strategies. OR Spectrum 27: 435-457.

Hopp, W., and M. Spearman. 1996. Factory Physics: Foundations of Factory Management. Hill Chicago, IL: Irwin/McGraw.

Karaesmen, F., J. A. Buzacott, and Y. Dallery. 2002. Integrating advance order information in make-to-stock production systems. IIE Transactions 34:649-662.

Krishnamurthy, A., and D. Claudio. 2005. Pull Systems with Advance Demand Information. In Proceeding of the 2005 Winter Simulation Conference, ed. M. E. Kuhl, N. M. Steiger, F. B. Armstrong, and J. A. Joines, 1733-1742. Piscataway, New Jersey: Institute of Electrical and Electronics Engineers, Inc. .

Krishnamurthy, A., R. Suri, and M. Vernon. 2004. Reexamining the Performance of MRP and Kanban Ma- terial Control Strategies for Multi-product Flexible Manufacturing Systems. International Journal of Flexible Manufacturing Systems 16: 123-150.

Liberopoulos, G., and S. Koukoumialos. 2005. Tradeoffs between base stocklevels, numbers of kanbans, and planned supply lead times in production/inventory systems with advance demand information. International Journal of Production Economics 96: 213-232.

Reiner, G., and M. Trcka. 2004. Customized supply chain design: Problems and alternatives for a production company in the food industry. A simulation based analysis. International Journal of Production Economics 89: 217-229

Zipkin, P.H., 2000. Foundations of Inventory Management. Boston, MA: McGrawHill.

\section{ACKNOWLEDGMENTS}

We would like to give special thanks to Dr. Deborah J. Medeiros for her lectures in AutoMod and for her close reading of an earlier version of this paper.

\section{AUTHOR BIOGRAPHIES}

DAVID CLAUDIO is a $\mathrm{PhD}$ student at the department of Industrial Engineering at the Pennsylvania State University. He has an MS in Industrial and Management Engineering from Rensselaer Polytechnic Institute. His interests include Human Factors, Service Systems, and Supply Chain Management. His e-mail address is <dave. claudio@psu.edu>

JIE ZHANG (JAY) is a PhD student at the department of Industrial Engineering at the Pennsylvania State University. His interests include Supply Chain Management, RFID, statistical analysis, etc. His e-mail address is <jxz208@psu.edu>

YING ZHANG (RENEE) is a M.S. student at the department of Industrial Engineering at the Pennsylvania State University. Her interests include Statistical analysis, Supply Chain Management, Information retrieval, etc. Her email address is <yzz114@psu. edu> 